\title{
Increased experience amplifies the activation of task-irrelevant category representations
}

\author{
Rachel Wu ${ }^{1} \cdot$ Zoe Pruitt $^{2} \cdot$ Benjamin D. Zinszer ${ }^{2}$. Olivia S. Cheung ${ }^{3}$
}

Published online: 15 December 2016

(C) The Psychonomic Society, Inc. 2016

\begin{abstract}
Prior research has demonstrated the benefits (i.e., task-relevant attentional selection) and costs (i.e., taskirrelevant attentional capture) of prior knowledge on search for an individual target or multiple targets from a category. This study investigated whether the level of experience with particular categories predicts the degree of task-relevant and task-irrelevant activation of item and category representations. Adults with varying levels of dieting experience (measured via 3 subscales of Disinhibition, Restraint, Hunger; Stunkard \& Messick, Journal of Psychosomatic Research, 29(1), 71$83,1985)$ searched for targets defined as either a specific food item (e.g., carrots), or a category (i.e., any healthy or unhealthy food item). Apart from the target-present trials, in the target-absent "foil" trials, when searching for a specific item (e.g., carrots), irrelevant items from the target's category (e.g., squash) were presented. The ERP (N2pc) results revealed that the activation of task-relevant representations (measured via Exemplar and Category N2pc amplitudes) did not differ based on the degree of experience. Critically, however, increased dieting experience, as revealed by lower Disinhibition scores, predicted activation of task-irrelevant representations (i.e., attentional capture of foils from the target item category). Our results suggest that increased experience with particular categories encourages the rapid activation of
\end{abstract}

Rachel Wu

Rachel.Wu@ucr.edu

1 Department of Psychology, University of California, Riverside, 900 University Avenue, Riverside, CA 92521, USA

2 Brain and Cognitive Sciences, University of Rochester, Rochester, NY, USA

3 Science Division, Psychology, New York University Abu Dhabi, Saadiyat Island, Abu Dhabi, United Arab Emirates category representations even when category information is task irrelevant, and that the N2pc in foil trials could potentially serve as an indication of experience level in future studies on categorization.

Keywords N2pc ERP · Attentional selection · Dieting · Categorization

Throughout our lifetime, we continuously acquire knowledge about objects and categories in the environment. How does prior experience shape our ability to search for familiar objects and categories when they are task relevant and ignore them when they are task irrelevant? Researchers have proposed that searching for a target requires an attentional "template," which is an activated and prioritized working memory representation of the target (Chelazzi, Miller, Duncan, \& Desimone, 1993; Olivers, Peters, Houtkamp, \& Roelfsema, 2011). This template can contain target information pertaining to individual features (e.g., the color blue; Eimer, 1996; Luck \& Hillyard, 1994), an object with multiple features (e.g., the blue numeral 2; Eimer \& Grubert, 2014), or even an object category (e.g., any numerals; Nako, Wu, \& Eimer, 2014; Wu et al., 2013). Successful search is likely due to the activation of appropriate target representations (i.e., attentional template), while suppressing distractor representations (Hodsoll \& Humphreys, 2001; Luck \& Hillyard, 1994; Sawaki \& Luck, 2010).

A frequently used method for testing the activation of target templates in a search task with human subjects is measuring the N2pc event-related potential (ERP). The N2pc component is the most established and earliest ERP marker of attentional target selection, emerging $200 \mathrm{~ms}$ after stimulus onset contralateral to the hemifield of the target (e.g., Eimer, 1996; Luck \& Hillyard, 1994). The N2pc was first discovered in studies using specific well-defined simple targets (e.g., a green 
vertical bar; Eimer, 1996; Luck \& Hillyard, 1994) to investigate aspects of attentional selection isolated from influences of prior knowledge. Many studies using the N2pc component have investigated whether the N2pc is a marker of top-down search or bottom-up capture (e.g., Eimer \& Kiss, 2010; Theeuwes, 2010), and the attentional factors that amplify or eliminate the N2pc, such as cueing effects (e.g., Woodman, Arita, \& Luck, 2009) and conjunction search (e.g., Luck, Chelazzi, Hillyard, \& Desimone, 1997).

More recent N2pc studies have investigated the role of prior knowledge in search by eliciting the component with complex familiar targets (e.g., letters and numerals, clothing, common household objects, animals, faces; Nako, Wu, \& Eimer, 2014; Nako, Wu, Smith, \& Eimer, 2014; Telling, Kumar, Meyer, \& Humphreys, 2009; Wu et al., 2015; Wu et al., 2013) or newly learned complex targets (e.g., aliens; $\mathrm{Wu}$, Pruitt, Runkle, Scerif, $\&$ Aslin, 2016). One of the most useful discoveries from N2pc studies using familiar objects as targets is that prior knowledge (e.g., knowledge that some items can be grouped into one category) impacts visual search via task-relevant and taskirrelevant activation of target representations. The amplitude of the N2pc is largest when there is only one target item to search for, and it decreases with an increasing number of different target items (Nako, Wu, \& Eimer, 2014). Interestingly, if participants are able to group target items into a single category (e.g., letters), the N2pc elicited by searching for any items in a target category also becomes similar to the N2pc elicited by the one-item search. In other words, the N2pc amplitude reflects the number of units in the target template, where one unit elicits the largest N2pc compared to 2+ units (after controlling for other factors, such as balanced search arrays). A significant $\mathrm{N} 2 \mathrm{pc}$ in a category search task reflects the activation and deployment of a unified template of different items in a category (Wu et al., 2016). The N2pc for category search has been observed for familiar complex naturalistic categories such as clothing, kitchen items, faces (Nako, Wu, Smith, et al., 2014; Wu et al., 2015), as well as newly learned categories, such as a family of alien characters (Wu et al., 2016).

In addition to the activation of task-relevant category representations, several ERP studies have also observed the activation of task-irrelevant category representations based on participants' prior knowledge about object categories (e.g., Nako, Wu, \& Eimer, 2014; Telling et al., 2009; see also Moores, Laiti, \& Chelazzi, 2003). During the "foil" trials in Nako et al. (2014), participants were instructed to search for one specific target object from a category (e.g., the letter $A$ in the Roman alphabet). However, instead of the target being present, another object from the same category (e.g., the letter $F$ ) appeared in the array, and participants were required to respond that the target was absent. An N2pc was observed during these target-absent trials with familiar objects from the same category as the target, indicating that the category representation may have been activated even though the category information was task-irrelevant for searching for a single target. Similar to the task-relevant category N2pc effect, the foil effects also have been observed for highly familiar categories (e.g., letters, numerals, clothing, kitchen items, faces). However, the foil effects have not been observed for newly trained categories (e.g., Chinese characters for nonChinese readers, Wu et al., 2013; novel alien characters, Wu et al., 2016).

Whereas it is clear that prior experience allows category templates to enhance task-relevant selection for any items from a category (i.e., benefit) and increases task-irrelevant capture when searching for a specific item and ignoring other items from the same category (i.e., cost), it is unclear whether the degree of prior experience predicts the activation of either or both task-relevant and task-irrelevant templates. It is important to note that, across studies, category N2pc effects (i.e., task-relevant activation) have been observed for highly familiar and novel categories, whereas foil N2pc effects (i.e., taskirrelevant activation) have only been observed for familiar categories. Therefore, it is possible that experience has a greater impact on task-irrelevant activation of category representations, compared to task-relevant activation of such information. A recent ERP study suggests that lifelong expertise with human faces (rather than ape faces) impacts only behavioral responses (i.e., perceptual narrowing; Scott, Pascalis, \& Nelson, 2007), while the N2pc effects for activation of representations of a single target and of a category are present for both human and ape faces, regardless of whether the category information is relevant or not (Wu et al., 2015). In other words, prior lifelong experience did not seem to impact the activation of item or category representations in task-relevant or task-irrelevant ways. The similar N2pc effects observed for human and ape faces challenges the unresolved issue regarding the extent to which the involuntary activation of category representations is influenced by firsthand experience with the categories. However, given the constraints of the study's stimuli (i.e., ape faces are too perceptually distinct from human faces to be included in the same N2pc experiment), the ape face search task and human face search task in Wu et al. (2015) were conducted in two separate experiments with different participants, rather than one experiment with the same participants. In addition, it was assumed (based on perceptual narrowing theories) that experience was greater for human faces and reduced for ape faces for all participants (and this assumption was confirmed in the behavioral responses) there was no independent measure of experience level, and experience level for both categories was assumed to be similar across participants. Therefore, only limited conclusions can be drawn about prior experience impacting the activation of task-relevant and task-irrelevant representations (as measured via the N2pc component), even though there is clear evidence of prior experience impacting search performance reflected in the behavioral responses. 
It has been shown in behavioral studies (in the same experiment with the same participants) that expertise with a taskirrelevant category (e.g., cars) may interfere with selecting task-relevant targets from a category that the observer also has expertise with (e.g., faces; McGugin, McKeeff, Tong, \& Gauthier, 2011). McGugin et al. (2011) also demonstrated that the amount of performance attenuation was correlated with the level of experience (e.g., greater expertise for cars was related to greater costs in search performance for faces when cars were distractors). Understanding the costs and benefits of the use of category representations provides insight into how prior knowledge impacts attentional selection.

This study investigated whether the degree of real-world experience predicts the degree of activation of target item representations and the degree of activation of task-relevant and task-irrelevant category representations. To study this issue, we used a visual search task that measured the N2pc with three requirements: (1) the use of two familiar categories that have high cross-category similarity, to avoid low-level bottom-up effects where categories could be defined by distinctive visual features, such as faces versus cars and ape faces versus human faces; (2) similar expertise within individuals in both categories to avoid asymmetric category effects; and (3) a quantifiable degree of expertise varying among individuals in both categories. We addressed all of these points by measuring the impact of dieting experience on the search for healthy versus unhealthy food. First, because healthy and unhealthy categories contain a broad variety of food items, we can include only items that are perceptually similar between categories (e.g., carrot sticks vs. Cheetos) in our search task. Second, dieting necessitates the awareness of categorical boundaries for both healthy and unhealthy food. Third, dieting experience can be quantified via the widely used Three Factor Eating Questionnaire (TFEQ; Stunkard \& Messick, 1985), which contains three subscales: Disinhibition, Restraint, and Hunger measures.

In the TFEQ dieting questionnaire (Stunkard \& Messick, 1985), Disinhibition refers to losing control of eating habits and is likely based on implicit and/or unconscious impulses. Restraint refers to conscious and explicit resistance to eating. Hunger refers to general food cravings or feelings of hunger. With the TEFQ, increased dieting experience is typically quantified by lower Disinhibition (decreased likelihood of binge eating), higher Restraint, and higher Hunger levels. Thousands of studies have linked the subscales in this dieting questionnaire to actual dieting outcomes, including weight gain and loss (e.g., Elfhag \& Rössner, 2005; Karlsson et al., 1994; Mcguire, Wing, Klem, \& Hill, 1999). Lower Disinhibition, specifically, has been shown to reliably predict the maintenance of weight loss (Fogelholm, Kukkonen-Harjula, \& Oja, 1999; see also Mcguire et al., 1999). Using the TEFQ (or a revised version) as a measure of dieting experience to examine biases and behavior, previous work has shown that people who diet more seem to be more sensitive to food-related information compared to people who diet less. For instance, dieters, who show high Restraint, are faster at finding food words among neutral words and neutral words among food words, compared to normal eaters who show low Restraint (Hollitt, Kemps, Tiggemann, Smeets, \& Mills, 2010; see also Fletcher, Pine, Woodbridge, \& Nash, 2007). However, after being primed with palatable foods, restrained dieters (but not unrestrained eaters) show more hedonic affect toward words for palatable/unhealthy foods (e.g., pizza) compared to neutral words (e.g., book; Hofmann, van Koningsbruggen, Stroebe, Ramanathan, \& Aarts, 2010). Along similar lines, restrained dieters under high cognitive load are more likely to overeat (i.e., display heightened Disinhibition) compared to unrestrained eaters (Mann \& Ward, 2004, 2007; Ward \& Mann, 2000). These examples suggest that Restraint relies on conscious and explicit mechanisms, while Disinhibition refers to more implicit, automatic processes.

Our study investigated whether dieting experience (as measured by the TEFQ) would impact activation of taskrelevant templates for single target food items and taskrelevant and task-irrelevant templates for healthy versus unhealthy food categories. Based on the dieting literature reviewed above, increased Restraint may lead to enhanced activation of task-relevant item and category representations. However, prior N2pc studies suggest that the amount of experience may not influence task-relevant activation (as measured by the N2pc on target-present trials) when searching for a single item or for any items in a category. Exemplar and category N2pc effects have been readily observed for both highly familiar and newlylearned objects (Nako, Wu, \& Eimer, 2014; Nako, Wu, Smith, et al., 2014; Wu et al., 2015; Wu et al., 2013; $\mathrm{Wu}$ et al., 2016). By contrast, activation of task-irrelevant category representations (as measured by the N2pc in "foil" trials) may be modulated by dieting experience, specifically decreased Disinhibition, because this construct reflects implicit responses to healthy and unhealthy food. This "foil N2pc" effect has only been observed previously for familiar stimuli (Nako, Wu, \& Eimer, 2014; Nako, Wu, Smith, et al., 2014; Telling et al., 2009; Wu et al., 2015; Wu et al., 2013), but not for less familiar newly learned complex stimuli (e.g., Chinese characters for non-Chinese readers, $\mathrm{Wu}$ et al., 2013; novel alien characters, Wu et al., 2016). While all three TFEQ subscales were included in this study, we expected that Disinhibition would affect task-irrelevant activation (i.e., foil N2pc), that Restraint may affect top-down explicit task-relevant activation of item and category representations, and that Hunger may globally affect activations of both task-relevant and task-irrelevant representations. 


\section{Method}

\section{Participants}

Eighteen participants $(M=22.39$ years, $\mathrm{S} E=0.90,11$ females, seven males) provided data for this study. They were recruited via a typical undergraduate subject pool at the University of Rochester and were not clinical dieters (i.e., no clinically diagnosed extreme eating habits or eating disorders). An additional three participants were excluded due to excessive eye movements in over $50 \%$ of ERP trials. All participants received $\$ 25$ for their time.

\section{Stimuli}

The full-color stimuli consisted of 12 images of healthy foods (squash, raisins, bananas, pears, onions, pumpkins, carrots, tomatoes, lemons, mushrooms, chickpeas, grapes) and 12 images of unhealthy foods (fries, chocolate, butter, fried chicken, cupcakes, croissants, Cheetos, steak, Mac ' $n$ Cheese, burgers, onion rings, bacon; see Fig. 1). These 24 images were selected from a total of 40 images of food items that the participants from a convenience sample $(N$ $=43$, mean age $=23.03$ years) consistently categorized $(>95 \%)$ as either healthy or unhealthy. Each item in the healthy category was visually similar to another item in the unhealthy category in terms of color, general shape, and/or size (e.g., carrots vs. Cheetos; peeled bananas vs. butter bricks). Specifically, there were no statistical differences between the healthy and unhealthy items overall, in terms of red-green-blue (RGB), hue-saturation-value/ brightness (HSV/B), lightness-saturation-hue (LCH), and lightness-red/green-yellow/blue (LAB) values, $t(22)<$ $1.15, p>.266$. Each item was isolated on a gray background (RGB: 96, 96, 96) and each image subtended $5.34^{\circ} \times 5.34^{\circ}$. Two items were presented in each search array, one on each side of the fixation point, to elicit the $\mathrm{N} 2 \mathrm{pc}$ from ipsilateral and contralateral electrode sites.

\section{Design and procedure}

Prior to the main experiment, participants completed a standard dieting questionnaire with Disinhibition, Restraint, and Hunger scales taken from Stunkard and Messick (1985). In general, increased dieting experience is reflected by lower Disinhibition, higher Restraint, and higher Hunger. To ensure the participants' ability to complete the visual search experiment, participants first categorized all food images to be shown in the main experiment in a pen-and-paper task: half of the participants circled all healthy food items, and the other half circled all unhealthy items. The participants were allowed to take as much time as they needed to complete this task accurately. The high accuracy in this categorization task (mean $=94 \%$,
$S D=5 \%$, range: $83 \%-100 \%$ ) confirmed that the food items were consistently categorized correctly. Prior to the main experiment, participants received feedback on the incorrectly categorized items and reviewed all of the items with the correct category labels simultaneously presented on a piece of paper.

In the main experiment, each participant completed three search tasks in a single experimental session: one exemplar search task (eight blocks of trials for either a specific healthy or unhealthy item) and two category search tasks (eight blocks of search trials for healthy food items and eight blocks for unhealthy food items), with task order counterbalanced across participants. The two category search tasks were combined in the analyses. ${ }^{1}$ For the exemplar search task, participants searched for a specific food item indicated at the beginning of the task. The specific food item in the exemplar search task was held constant for the entire duration of the experiment for each participant, because previous studies showed that it is extremely difficult for the participant to switch targets on every trial (e.g., Wu et al., 2013). Across participants, all exemplars were assigned as the search targets to at least one participant. For the two category search tasks, participants searched in separate tasks for either all healthy or all unhealthy food items.

In the exemplar search task, each of the eight blocks consisted of 28 exemplar match trials (e.g., the target healthy food item appeared, along with an unhealthy food item), 28 foil trials (e.g., a non-target healthy food item appeared, along with an unhealthy item), and six no target trials (e.g., two unhealthy items appeared; see Fig. 2). To balance the number of target-present and target-absent responses in the exemplar search task and to maximize the number of foil trials, we included the same number of exemplar match and foil trials, while including a few no target trials. The presentation order of all trial types was randomized. The number of trials per trial type in this study was the same as in previous studies with a similar design (e.g., Wu et al., 2015; Wu et al., 2016). Each of the eight category search blocks per task (i.e., healthy and unhealthy category search tasks) contained 28 category match trials (e.g., any of the healthy food items appeared on one side of the array with any of the unhealthy items on the other side of the array) and 28 no target trials (e.g., two unhealthy items appeared in the array in the healthy category search task). There were no foil trials in the

\footnotetext{
${ }^{1}$ To reduce fatigue in the participants and to maximize the number of trials per task, we only included one (instead of two) exemplar search task and two category search tasks. Therefore, an item from one category was included as the target for two tasks, while items from the other category were the target for only one task. By doing so, we may have enhanced the efficiency for searching for one of the categories. However, the assignment of participants to the task conditions was counterbalanced and random, and the neural and behavioral responses to both categories were averaged within participants. Therefore, although the design did not support comparing within subjects healthy versus unhealthy search trials, it accounted for the imbalance in target relevance across participants.
} 

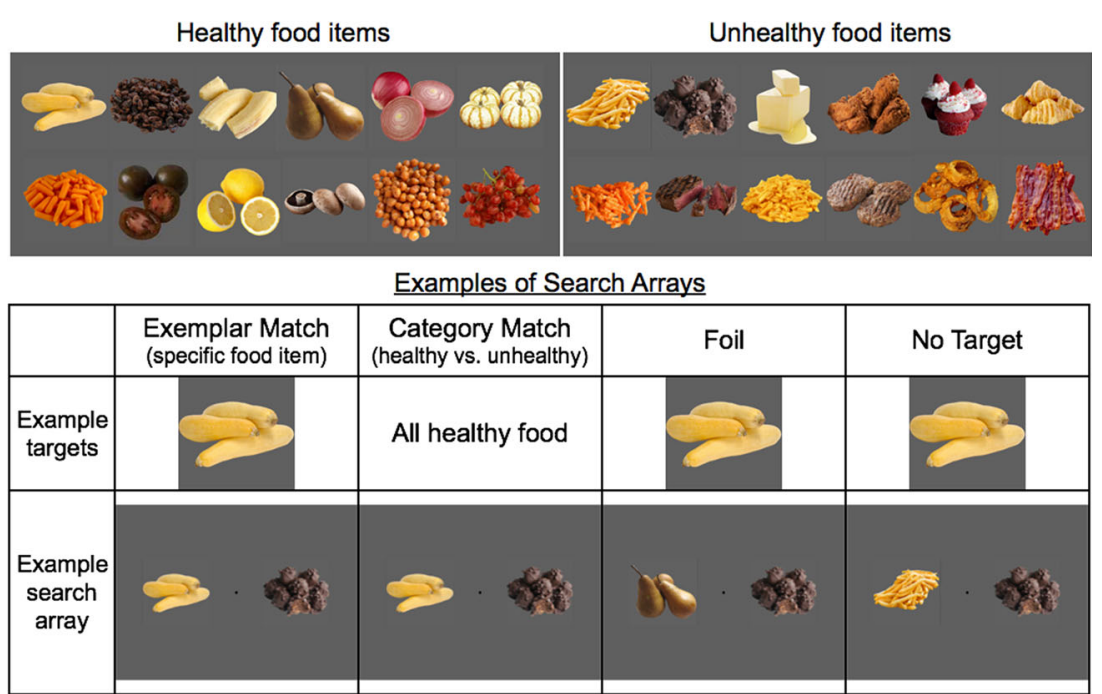

Fig. 1 Healthy and unhealthy food items used as search stimuli (top panel). Example search arrays from target (exemplar or category match trials), foil, and no target trials (bottom panel)

category search blocks, as all items from the target category were considered targets in this task. The presentation order of the category match and no target trials was randomized. In the entire experimental session, there were 1,392 trials in total.

Each two-item search array was displayed for $200 \mathrm{~ms}$, followed by a response window of 1,600 ms that displayed only the small black fixation dot in the center of the gray screen (see Fig. 2). Participants indicated target presence or absence by pressing either the left or right arrow keys. Participants were instructed to answer as quickly and as accurately as possible and to blink only during intertrial intervals after responding, while maintaining fixation on the central dot throughout the experiment.

\section{EEG recording and data analysis}

At standard positions of the extended 10/20 system, the EEG was DC recorded from 32 scalp electrodes with a $500 \mathrm{~Hz}$ sampling rate and off-line $40 \mathrm{~Hz}$ low-pass filter. The data were recorded with a left earlobe reference and re-referenced off-line to the averaged earlobes. We used a 100 -ms prestimulus baseline for epochs from $-100 \mathrm{~ms}$ to $500 \mathrm{~ms}$ relative to the search array onset. For the artifact rejection criteria, we used horizontal EOG exceeding $\pm 25 \mu \mathrm{V}$, vertical EOG exceeding $\pm 60 \mu \mathrm{V}$, all other channels exceeding $\pm 80 \mu \mathrm{V}$. For all ERP analyses, only correct trials were used. After eye-movement artifact rejection, we retained $73 \%$ of all correct trials on average per participant. The time window used to determine the mean N2pc amplitude

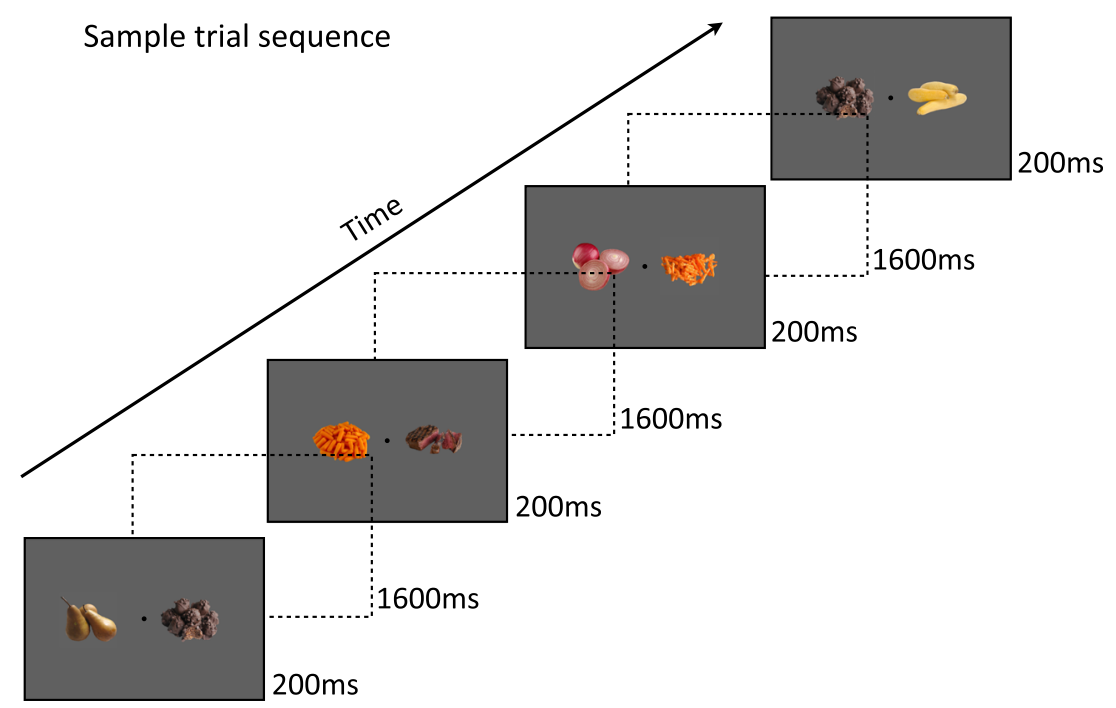

Fig. 2 Sample sequence of trials in both exemplar and category search tasks 

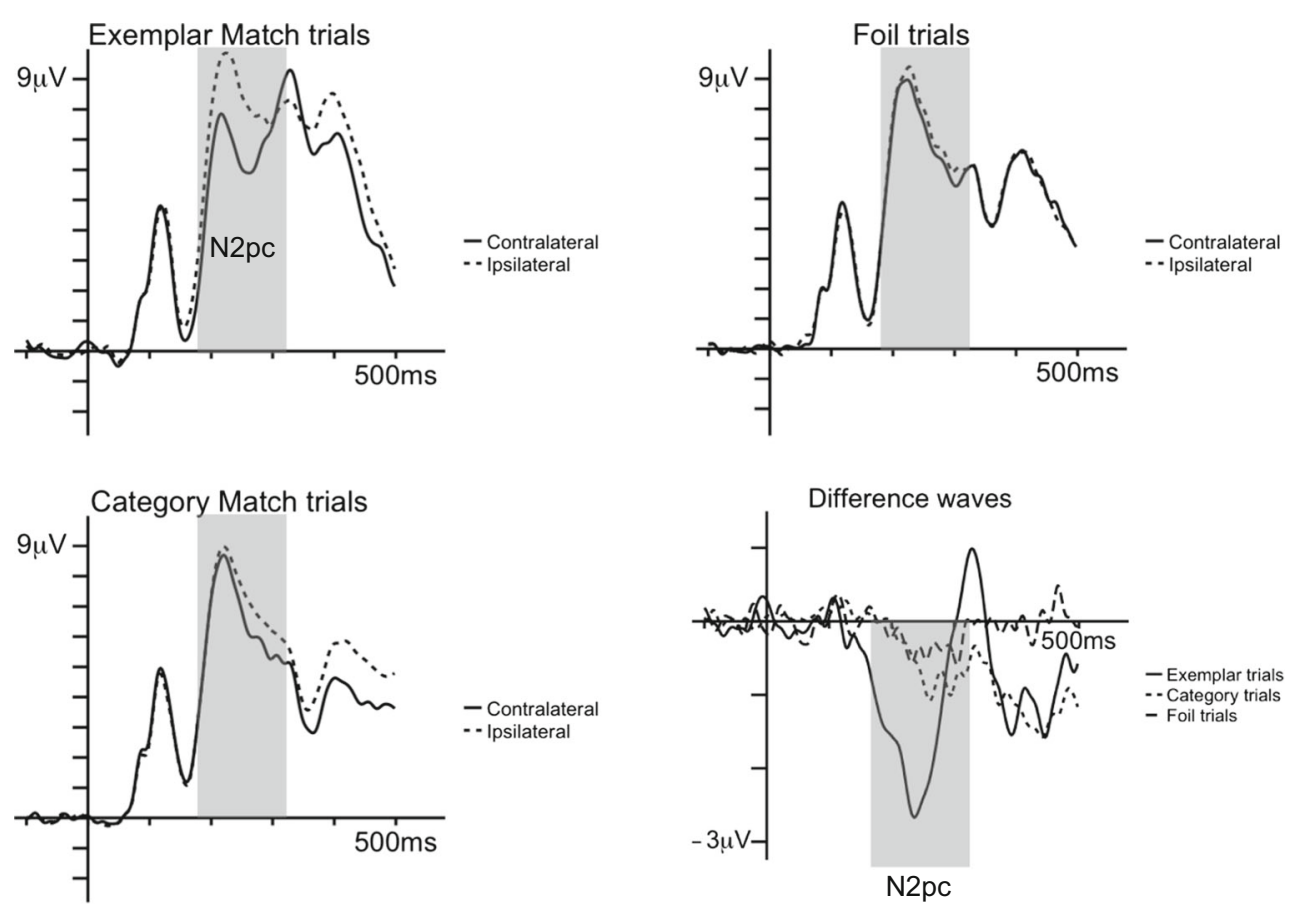

Fig. 3 Grand average ERPs elicited in response to search arrays for exemplar, category match, and foil trials at posterior electrodes PO7/8 contralateral and ipsilateral to a target item. N2pc difference waveforms

were obtained by subtracting ipsilateral from contralateral ERP waveforms at $\mathrm{PO} 7 / 8$ for each trial type

was $180 \mathrm{~ms}$ to $320 \mathrm{~ms}$ after search array onset at lateral posterior electrodes PO7 and PO8 (Nako, Wu, \& Eimer, 2014).

\section{Results}

This study investigated whether the degree of dieting experience predicts the degree of activation of task-relevant single target representations and the degree of activation of taskrelevant and task-irrelevant category representations. In this study, task-relevant activation was quantified by the N2pc amplitude on target-present trials (i.e., exemplar match and category match), while task-irrelevant activation was quantified by the N2pc amplitude on target-absent trials in the exemplar search tasks (i.e., foil trials). The no target trials (combined from both the category search task and exemplar search task) could not be included in the ERP analyses due to the contralateral nature of the $\mathrm{N} 2 \mathrm{pc}$ : Referencing a target or foil in a specific location is required for N2pc analyses to specify whether $\mathrm{PO} 7$ or PO8 is the ipsilateral or contralateral electrode on a given trial. Following previous studies (e.g., Nako, Wu, \& Eimer, 2014; Nako, Wu, Smith, et al., 2014; Wu et al., 2015; Wu et al., 2016), the ERP analyses included the target-present trials (exemplar match, category match) and only foil trials for target-absent trials, while the behavioral analyses included target-present trials (exemplar match and category match) and target-absent trials (foil and no target). The ERP (N2pc) results support the hypothesis that increased

experience is related to increased activation of task-irrelevant category representations. Next, we report in detail the ERP results on task-relevant and task-irrelevant activation of attentional templates and the relation to dieting experience, and include the behavioral analyses on the overall performance in the visual search task.

\section{ERP results}

For the analyses of the ERP data, we first investigated the presence of and differences in N2pc amplitude among exemplar match, category match, and foil trials, and then tested whether the degree of dieting experience predicted the N2pc amplitude in the different trial types. The no target trials were not included in these analyses since there was no preidentified target or foil for those trials.

Overall N2pc amplitude The N2pc difference wave between contralateral and ipsilateral waveforms was evaluated for exemplar match, category match, and foil trials. The results are illustrated in Figs. 3 and 4. A 2 (laterality) $\times 3$ (trial type) ANOVA confirmed a significant main effect of laterality, $F(1,17)=22.52, p<.001, \eta^{2}=0.57$, and a significant interaction between laterality and trial type, $F(2,34)=2.24, p=$ $.002, \eta^{2}=0.31$. Pairwise $t$ tests (adjusted $\alpha=0.017$ ) between contralateral and ipsilateral mean amplitudes revealed significant N2pc components for exemplar match trials, $t(17)=$ $4.38, p<.001$, and category match trials, $t(17)=3.25, p=$ 
.005 , but the N2pc for foil trials only approached significance, $t(17)=1.79, p=.092$. Pairwise $t$ tests (adjusted $\alpha=0.017$; see Fig. 4) revealed that the N2pc for exemplar match trials was larger than the N2pc for foil trials, $t(17)=3.36, p=.004$, and marginally larger than the N2pc for category match trials, $t(17)=2.56, p=.020$, and there was no difference between foil and category match trials, $t(17)=1.17, p=.258$. These results are entirely consistent with previous studies using a similar paradigm (e.g., Nako et al., 2014a, b; Wu et al., 2015; Wu et al., 2016), as the N2pc is typically most robust for task-relevant cases, and is larger when the search involves a single target item compared to multiple target items from the same category. The N2pc observed for foil trials, which indicates activation of task-irrelevant category representations, but is often weaker than the N2pc for target relevant searches, was only marginally significant here. Below, we further examined whether the significant N2pc effect for exemplar match and category match trials, and the marginally significant N2pc effect for foil trials, might be modulated by dieting experience based on our prior hypotheses that Disinhibition would affect activation in target-absent trials (foil N2pc), that Restraint may affect activation in target-present trials (exemplar and category $\mathrm{N} 2 \mathrm{pc}$ ), and that Hunger may globally affect both targetpresent and target-absent trials.

Dieting experience and N2pc To analyze the effects of the dieting experience scores on the N2pc amplitude for each trial type, we estimated two separate regression models for the target-present trials (i.e., exemplar match and category match) and target-absent trials (i.e., foil trials). For the target-present trials (i.e., exemplar match and category match trials), no dieting measures significantly predicted the N2pc amplitudes for either of the target-present trial types, $t(17)<.51$, revealing no influence of dieting experience on these trials. However, for the Foil trials, while no significant effect was found for the Hunger and Restraint scales on the N2pc component, Disinhibition ratings significantly predicted N2pc amplitude, $t(17)=2.70, p=.018, \beta=0.629$ : Specifically, a lower Disinhibition rating (i.e., increased dieting experience) predicted a larger foil N2pc amplitude (i.e., a larger negative amplitude; see Fig. 5), revealing the influence of dieting

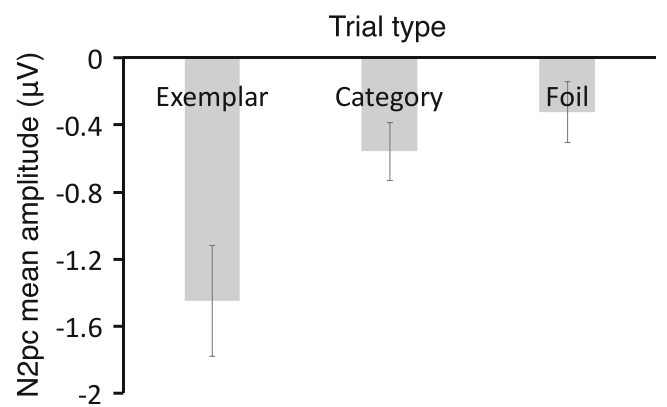

Fig. 4 N2pc mean amplitudes from exemplar, category, and foil trials. Error bars represent $\pm 1 S E$

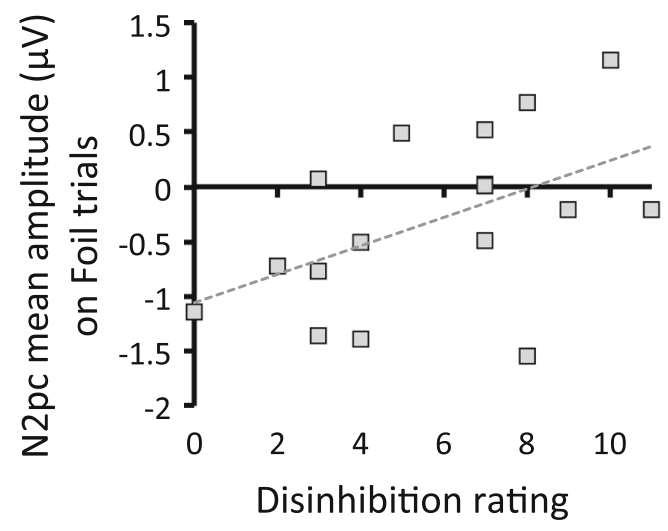

Fig. 5 Disinhibition ratings from the dieting questionnaire (TEFQ; Stunkard \& Messick, 1985) predicted the N2pc mean amplitude on foil trials

experience on the activation of irrelevant category information during the search for a single target. ${ }^{2}$

\section{Behavioral results}

For the behavioral results, we investigated the differences in accuracy and reaction time among the four trial types. A oneway ANOVA with the within-subject factor trial type (exemplar/category/foil/no target) was conducted over logtransformed reaction times (RT) and accuracy (see Fig. 6). There was a significant difference among trial type, RT: $F(3$, $51)=150.79, p<.001, \eta^{2}=0.90$, Accuracy: $F(3,51)=11.65$, $p<.001, \eta^{2}=0.41$. Planned $t$ tests were then conducted separately on target-present trials (exemplar match vs. category match) and target-absent trials (foil vs. no target) (adjusted $\alpha=0.025$ ) to analyze differences between trial types requiring the same response. For the target-present trials, performance was faster and better for exemplar match than category match, RT: $t(17)=13.35, p<.001$, accuracy $t(17)=4.72, p<.001$. This result is consistent with previous findings that it is easier to search for an exact target than for multiple possible items from a category. By contrast, for the target-absent trials, RT was faster but accuracy was worse for foil than no target trials, RT: $t(17)=13.07, p<.001$, accuracy: $t(17)=-2.73, p=.014$. Although we should not overinterpret this result because of the speed-accuracy trade-off, this result appeared to be consistent with the idea that category representations might have been activated even when participants searched for only one target, and thus the presence of a foil might prompt the participants to respond quickly but incorrectly.

\footnotetext{
${ }^{2}$ Note that we also explored whether the accuracy of the categorization quiz (taken prior to the study) might predict the ERP results. The quiz score predicted the category N2pc amplitude at marginal significance, $t(17)=2.03, p=$ $.063, \beta=-0.480$. On the other hand, dieting experience levels did not predict the category quiz scores, perhaps due to ceiling effects on the categorization quiz.
} 

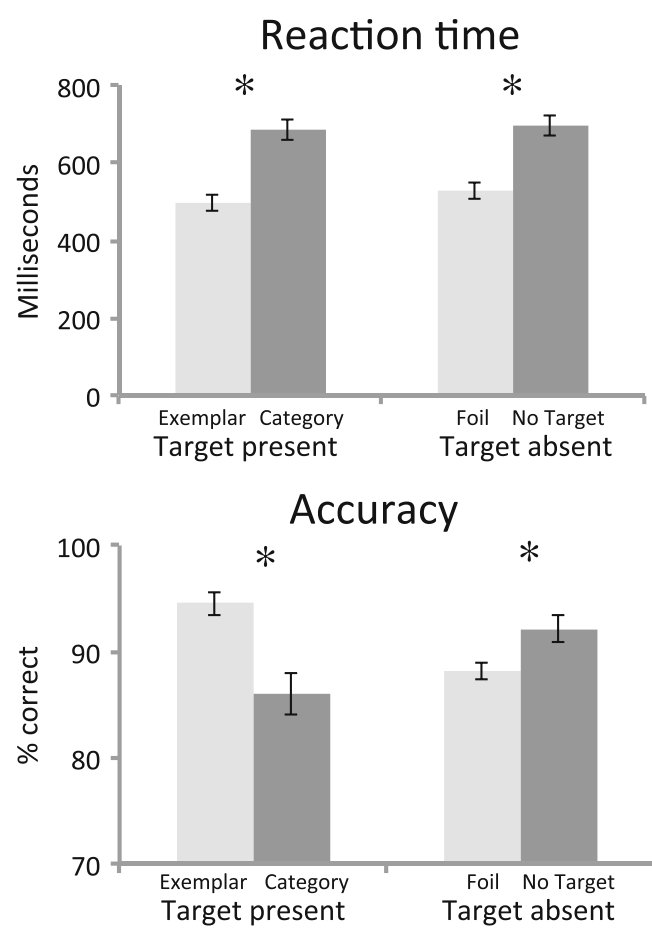

Fig. 6 Reaction time and accuracy for all trial types split by targetpresent and target-absent responses. Error bars represent $\pm 1 S E$. ${ }^{*} p<.015$

\section{Discussion}

The present ERP study investigated whether the degree of experience (i.e., dieting) impacts the activation of taskrelevant item representations and the activation of taskrelevant and task-irrelevant category representations when participants searched for either a specific item or a category. Dieting experience was quantified by a standard dieting questionnaire that included three subscales: Disinhibition, Restraint, and Hunger (Stunkard \& Messick, 1985). Taskrelevant activation of a single target representation and of a category representation (i.e., item and category attentional templates) was quantified via $\mathrm{N} 2 \mathrm{pc}$ amplitudes during exemplar and category match trials, whereas activation of a task-irrelevant category representation when searching for a single target was quantified via N2pc amplitudes during foil trials. As predicted, our ERP results revealed that increased dieting experience differentially impacted the activations of task-relevant versus task-irrelevant attentional templates. Specifically, significant N2pc effects were observed for task-relevant representations for either a single target food item or a target category of food items regardless of experience level. However, increased dieting experience, as indicated by lower scores in the Disinhibition scale, which represents decreased implicit loss of control over eating habits (e.g., overeating), predicted larger foil N2pc amplitudes, compared to decreased dieting experience. This finding suggests that when participants searched for a specific food item (e.g., carrots) and were supposed to ignore other food items from the same category (e.g., other healthy food items), a task-irrelevant category representation (e.g., healthy food) was more likely to be activated in more experienced rather than less experienced participants. By contrast, the less experienced observers appeared to be less distracted by non-target members from the same category as the target when searching for a specific food item.

Our ERP results are novel and important for two main reasons. First, we demonstrated that the degree of prior experience predicts the amount of activation of task-irrelevant representations. Most N2pc studies thus far have focused on how search is guided by attentional factors, such as top-down (goal-directed) or bottom-up (stimulus-driven) effects (e.g., Eimer \& Kiss, 2010; Theeuwes, 2010), cueing effects (e.g., Woodman et al., 2009), or multiple features (e.g., Luck et al., 1997), using "pure" simple stimuli that are not supposed to be influenced by prior knowledge. An increasing number of studies on visual search and working memory show that prior knowledge (e.g., statistical relations, category knowledge) can allow us to overcome attentional and memory limitations by grouping or "compressing" information (e.g., Brady, Konkle, \& Alvarez, 2009; Brady, Störmer, \& Alvarez, 2016; Wu et al., 2016; see also Yang \& Zelinsky, 2009). The use of prior knowledge also seems to come at a cost, such as semantic interference in visual search (e.g., Telling et al., 2009) or false alarms to foil non-target items within the target's category (e.g., selecting the letter $R$ when the letter $A$ is the target; Nako, Wu, \& Eimer, 2014). Previous N2pc studies have suggested that these costs may be due to inappropriate target templates developed for familiar stimuli: significant foil N2pc amplitudes have been elicited only for highly familiar categories rather than novel categories (e.g., clothing vs. aliens; Nako et al., 2014b; Wu et al., 2013; Wu et al., 2015). Extending previous work, the present study demonstrated that the foil $\mathrm{N} 2 \mathrm{pc}$ amplitude depends on the degree of the experience: Increased dieting experience induces a $\mathrm{n}$ automatic activation of category representations of healthy and unhealthy food items, even when category information is task-irrelevant. The use of "inappropriate," or task-irrelevant, templates in this study coincides with other studies showing that when information at hand deviates from the statistics in the familiar natural environment, participants make more mistakes (Blanco et al., 2016; Green, Benson, Kersten, \& Schrater, 2010; Orhan, Sims, Jacobs, \& Knill, 2014). Naturally, adults have a strong tendency to rely initially on prior experience and employ familiar cognitive models and heuristics to solve various tasks. Such models and heuristics are adaptive in everyday life, but may be inappropriate for a current experimental task. Our study found that prior experience requiring the use of category boundaries (e.g., dieting) led observers to rapidly apply category knowledge to guide attention, even when the category information was task-irrelevant. Critically, the magnitude of 
the activation of such task-irrelevant representations appears to depend on the level of experience.

Second, the stimulus sets that we employed revealed that distinctive perceptual features among category members are not necessary to guide search, and that perceptually different items grouped into broad subjective categories also can guide search rapidly. In previous studies, the N2pc typically had been elicited with specific well-defined targets (e.g., the letter $A$ or a green diamond), and has only recently been shown to be elicited with a range of items from various finite or welldefined categories (e.g., any letter, any clothing; Nako, Wu, \& Eimer, 2014; Nako, Wu, Smith, et al., 2014; Telling et al., 2009; Wu et al., 2013; Wu et al., 2015; Wu et al., 2016). Previous categorization studies typically implement stimuli that are perceptually similar within a category (e.g., faces, cars, teddy bears; Freedman, Riesenhuber, Poggio, \& Miller, 2001; McGugin et al., 2011; Yang \& Zelinsky, 2009) or finite with strict boundaries (e.g., letters vs. numbers; Nako et al., 2014a). By contrast, heathy and unhealthy foods can be perceptually dissimilar within categories and similar between categories. Moreover, the category boundary between healthy and unhealthy food often varies due to subjectivity (e.g., "Is pizza a vegetable?"). While the healthy vs. unhealthy distinction might be subjective, the present visual search task provided consistent and clear category boundaries for all participants. Indeed, the participants in this study did not have to be on a diet to categorize the items correctly, which was exemplified by the high accuracy on the category quiz, indicating that all participants in this study largely agreed on the category boundary. The current study is the first to show that even diverse subjective categories can trigger a reliable N2pc ERP component, although the N2pc component may be attenuated when the category is broad and not easily well-defined perceptually or conceptually, as in this study.

In future work, the effect of experience on attentional templates should be further investigated by examining the type and amount of training/experience that would lead to the automatic activation of task-relevant and task-irrelevant category representations. Focusing on dieting experience as a means to study this question, our study used a common standardized dieting questionnaire to assess general dieting experience, which was inferred from undergraduates who were not preselected based on dieting behavior. Future work could incorporate additional complementary measures of dieting experience and dieting behavior, such as types of diets, successful or unsuccessful weight loss, and the reward value of healthy versus unhealthy foods, to accommodate the wide range of diets and dieting behaviors (e.g., in the Paleo diet, steak is "healthy," but it was in the "unhealthy" category in the present study). For example, successful and unsuccessful dieters have been shown to have different representations of healthy and unhealthy foods: After being primed with a palatable/unhealthy food item (e.g., chocolate muffin), a healthy food item (e.g., apple) seemed larger to successful dieters, but smaller to unsuccessful dieters relative to other unhealthy foods (van Koningsbruggen, Stroebe, \& Aarts, 2011). Successful dieting can depend on whether unhealthy food is seen as desirable/tempting or related to weight gain and therefore something to avoid (van Koningsbruggen, Stroebe, \& Aarts, 2013). These additional measures may provide further insights into the kind and/or amount of experience that might impact task-relevant and task-irrelevant activation of attentional templates in visual search in nonclinical dieters. Differences in attentional biases to food also have been observed with clinical populations, such as obese individuals, which may impact healthy food choices in general (e.g., Gearhardt, Treat, Hollingworth, \& Corbin, 2012). Patients with anorexia nervosa or bulimia nervosa have heightened top-down control when viewing food images (e.g., Brooks et al., 2011), which originate from frontal-striatal networks that underlie habitual behavior (Foerde, Steinglass, Shohamy, \& Walsh, 2015). Further investigation on the effects of learned biases (e.g., value judgments; category boundaries) on attentional selection may provide implications for specialized interventions. Also, given the small sample size for a study investigating a continuous predictor, these results should be replicated, especially with a larger sample size. The effect of prior knowledge on attentional guidance should also be generalized to other types of experience.

Investigations on how and why degree of experience benefits and hinders attentional selection is crucial for cognitive science, and dieting experience appears to be one appropriate means for such investigations. In general, it is important to understand how and why prior experience influences attentional selection because it has cascading effects on future learning (see Scerif, 2010). The current study showed that increased experience amplifies the activation of taskirrelevant category representations, which suggests that prior experience with categories may encourage the increased likelihood in retrieving category knowledge that can influence early attentional selection. Note that while such a cognitive model is highly efficient in familiar environments, it could be inefficient in novel environments or altered environments. Therefore, to what extent overlearned information may be flexibly retrieved or suppressed in familiar or altered/novel environments should be further explored. Finally, future research should clarify how and why prior experience impacts subsequent attention and learning to better understand tradeoffs and cascading effects of flexible versus inflexible cognitive abilities.

Acknowledgements We thank Richard Aslin and Rebecca Nako for useful discussions on the study design and data interpretations. We also thank Stefan Van der Stigchel, Veronica Mazza, and two other anonymous reviewers, as well as the members of the CALLA lab, for insightful comments on previous versions of the manuscript. This research was conducted at the University of Rochester with funding from an NRSA 
(F32HD070537) from NICHD to R.W., and from an NIH Grant (HD037082) to Richard Aslin.

\section{References}

Blanco, N. J., Love, B. C., Ramscar, M., Otto, A. R., Smayda, K., Blanco, N. J., ... Maddox, W. T. (2016). Exploratory decision-making as a function of lifelong experience, not cognitive decline. Journal of Experimental Psychology: General Experience, 145(3), 284-297.

Brady, T. F., Konkle, T., \& Alvarez, G. A. (2009). Compression in visual working memory: Using statistical regularities to form more efficient memory representations. Journal of Experimental Psychology: General, 138(4), 487-502. doi:10.1037/a0016797

Brady, T. F., Störmer, V. S., \& Alvarez, G. A. (2016). Working memory is not fixed-capacity: More active storage capacity for real-world objects than for simple stimuli. Proceedings of the National Academy of Sciences, 113(27), 7459-7464. doi:10.1073/pnas.1520027113

Brooks, S. J., O’Daly, O. G., Uher, R., Friederich, H.-C., Giampietro, V., Brammer, M., ... Campbell, I. C. (2011). Differential neural responses to food images in women with bulimia versus anorexia nervosa. PLOS ONE, 6(7), e22259. doi:10.1371/journal. pone. 0022259

Chelazzi, L., Miller, E., Duncan, J., \& Desimone, R. (1993). A neural basis for visual search in inferior temporal cortex. Nature, 363(6427), 345-347. doi:10.1038/363345a0

Eimer, M. (1996). The N2pc component as an indicator of attentional selectivity. Electroencephalography and Clinical Neurophysiology, 99(3), 225-234. doi:10.1016/S0921-884X(96)95711-2

Eimer, M., \& Grubert, A. (2014). Spatial attention can be allocated rapidly and in parallel to new visual objects. Current Biology, 24(2), 193-198. doi:10.1016/j.cub.2013.12.001

Eimer, M., \& Kiss, M. (2010). The top-down control of visual selection and how it is linked to the N2pc component. Acta Psychologica, 135(2), 100-102. doi:10.1016/j.actpsy.2010.04.010

Elfhag, K., \& Rössner, S. (2005). Who succeeds in maintaining weight loss? A conceptual review of factors associated with weight loss maintenance and weight regain. Obesity Reviews: An Official Journal of the International Association for the Study of Obesity, 6(1), 67-85. doi:10.1111/j.1467-789X.2005.00170.x

Fletcher, B. C., Pine, K. J., Woodbridge, Z., \& Nash, A. (2007). How visual images of chocolate affect the craving and guilt of female dieters. Appetite, 48(2), 211-217. doi:10.1016/j.appet.2006.09.002

Foerde, K., Steinglass, J. E., Shohamy, D., \& Walsh, B. T. (2015). Neural mechanisms supporting maladaptive food choices in anorexia nervosa. Nature Neuroscience. doi:10.1038/nn.4136

Fogelholm, M., Kukkonen-Harjula, K., \& Oja, P. (1999). Eating control and physical activity as determinants of short-term weight maintenance after a very-low-calorie diet among obese women. International Journal of Obesity, 23, 203-210.

Freedman, D. J., Riesenhuber, M., Poggio, T., \& Miller, E. K. (2001). Categorical representation of visual stimuli in the primate prefrontal cortex. Science, 291(5502), 312. Retrieved from http://www. sciencemag.org/content/291/5502/312.short

Gearhardt, A. N., Treat, T. A., Hollingworth, A., \& Corbin, W. R. (2012). The relationship between eating-related individual differences and visual attention to foods high in added fat and sugar. Eating Behaviors, 13(4), 371-374. doi:10.1016/j.eatbeh.2012.07.004

Green, C. S., Benson, C., Kersten, D., \& Schrater, P. (2010). Alterations in choice behavior by manipulations of world model. Proceedings of the National Academy of Sciences, 107(37), 16401-16406. doi:10.1073/pnas.1001709107

Hodsoll, J., \& Humphreys, G. W. (2001). Driving attention with the top down: The relative contribution of target templates to the linear separability effect in the size dimension. Perception \& Psychophysics, 63(5), 918-926. doi:10.3758/BF03194447

Hofmann, W., van Koningsbruggen, G. M., Stroebe, W., Ramanathan, S., \& Aarts, H. (2010). As pleasure unfolds: Hedonic responses to tempting food. Psychological Science, 21(12), 1863-1870. doi:10.1177/0956797610389186

Hollitt, S., Kemps, E., Tiggemann, M., Smeets, E., \& Mills, J. S. (2010). Components of attentional bias for food cues among restrained eaters. Appetite, 54(2), 309-313. doi:10.1016/j.appet.2009.12.005

Karlsson, J., Hallgren, P., Kral, J., Lindroos, A.-K., Sjostrom, L., \& Sullivan, M. (1994). Predictors and effects of long-term dieting on mental well-being and weight loss in obese women. Appetite, 23, $15-26$.

Luck, S. J., Chelazzi, L., Hillyard, S. A., \& Desimone, R. (1997). Neural mechanisms of spatial selective attention in areas V1, V2, and V4 of macaque visual cortex. Journal of Neurophysiology, 77(1), 24-42.

Luck, S. J., \& Hillyard, S. A. (1994). Spatial filtering during visual search: Evidence from human electrophysiology. Journal of Experimental Psychology: Human Perception and Performance. doi:10.1037/0096-1523.20.5.1000

Mann, T., \& Ward, A. (2004). To eat or not to eat: Implications of the attentional myopia model for restrained eaters. Journal of Abnormal Psychology, 113(1), 90-98. doi:10.1037/0021-843X.113.1.90

Mann, T., \& Ward, A. (2007). Attention, self-control, and health behaviors. Current Directions in Psychological Science, 16(5), 280-283.

McGugin, R. W., McKeeff, T. J., Tong, F., \& Gauthier, I. (2011). Irrelevant objects of expertise compete with faces during visual search. Attention, Perception \& Psychophysics, 73(2), 309-317. doi:10.3758/s13414-010-0006-5

Mcguire, M. T., Wing, R. R., Klem, M. L., \& Hill, J. O. (1999). What predicts weight regain in a group of successful weight losers ? Journal of Consulting and Clinical Psychology, 67(2), 177-185.

Moores, E., Laiti, L., \& Chelazzi, L. (2003). Associative knowledge controls deployment of visual selective attention. Nature Neuroscience, 6(2), 182-189. doi:10.1038/nn996

Nako, R., Wu, R., Smith, T. J., \& Eimer, M. (2014). Item and categorybased attentional control during search for real-world objects: Can you find the pants among the pans? Journal of Experimental Psychology. Human Perception and Performance, 40(4), 12831288. Retrieved from http://www.ncbi.nlm.nih. gov/pubmed $/ 24820441$

Nako, R., Wu, R., \& Eimer, M. (2014). Rapid guidance of visual search by object categories. Journal of Experimental Psychology: Human Perception and Performance, 40(1), 50-60. doi:10.1037/a0033228

Olivers, C. N. L., Peters, J., Houtkamp, R., \& Roelfsema, P. R. (2011). Different states in visual working memory: When it guides attention and when it does not. Trends in Cognitive Sciences, 15(7), 327-334. doi:10.1016/j.tics.2011.05.004

Orhan, A. E., Sims, C. R., Jacobs, R. A., \& Knill, D. C. (2014). The adaptive nature of visual working memory. Current Directions in Psychological Science, 23(3), 164-170. doi:10.1177 /0963721414529144

Sawaki, R., \& Luck, S. J. (2010). Capture versus suppression of attention by salient singletons: Electrophysiological evidence for an automatic attend-to-me signal. Attention, Perception \& Psychophysics, 72(6), 1455-1470. doi:10.3758/APP

Scerif, G. (2010). Attention trajectories, mechanisms and outcomes: At the interface between developing cognition and environment. Developmental Science, 13, 805-812. doi:10.1111/j.14677687.2010.01013.x

Scott, L. S., Pascalis, O., \& Nelson, C. A. (2007). A domain-general theory of the development of perceptual discrimination. Current Directions in Psychological Science, 16(4), 197-201. doi:10.1111 j.1467-8721.2007.00503.x

Stunkard, A. J., \& Messick, S. (1985). The three-factor eating questionnaire to measure dietary restraint, disinhibition and hunger. Journal 
of Psychosomatic Research, 29(1), 71-83. doi:10.1016/0022-3999 (85) $90010-8$

Telling, A. L., Kumar, S., Meyer, A. S., \& Humphreys, G. W. (2009). Electrophysiological evidence of semantic interference in visual search. Journal of Congitive Neuroscience, 22(10), 2212-2225. doi:10.1162/jocn.2009.21348

Theeuwes, J. (2010). Top-down and bottom-up control of visual selection. Acta Psychologica, 135(2), 77-99. doi:10.1016/j. actpsy.2010.02.006

van Koningsbruggen, G. M., Stroebe, W., \& Aarts, H. (2011). Through the eyes of dieters: Biased size perception of food following tempting food primes. Journal of Experimental Social Psychology, 47(2), 293-299. doi:10.1016/j.jesp.2010.10.012

van Koningsbruggen, G. M., Stroebe, W., \& Aarts, H. (2013). Successful restrained eating and trait impulsiveness. Appetite, 60, 81-84. doi:10.1016/j.appet.2012.09.016

Ward, A., \& Mann, T. (2000). Don't mind if I do: Disinhibited eating under cognitive load. Journal of Personality and Social Psychology, 78(4), 75-763.
Woodman, G. F., Arita, J. T., \& Luck, S. J. (2009). A cuing study of the N2pc component: An index of attentional deployment to objects rather than spatial locations. Brain Research, 1297, 101-111. doi:10.1016/j.brainres.2009.08.011

Wu, R., Nako, R., Band, J., Pizzuto, J., Ghoreishi, Y., Scerif, G., \& Aslin, R. (2015). Rapid attentional selection of non-native stimuli despite perceptual narrowing. Journal of Cognitive Neuroscience, 27(11), 2299-2307. doi:10.1162/jocn a 00857

Wu, R., Pruitt, Z., Runkle, M., Scerif, G., \& Aslin, R. N. (2016). A neural signature of rapid category-based target selection as a function of intraitem perceptual similarity, despite inter-item dissimilarity. Attention, Perception, \& Psychophysics.. doi:10.3758/s13414-015-1039-6

Wu, R., Scerif, G., Aslin, R. N., Smith, T. J., Nako, R., \& Eimer, M. (2013). Searching for something familiar or novel: Top-down attentional selection of specific items or object categories. Journal of Cognitive Neuroscience, 25(5), 719-729. doi:10.1162/jocn

Yang, H., \& Zelinsky, G. J. (2009). Visual search is guided to categorically-defined targets. Vision Research, 49(16), 2095-2103. doi:10.1016/j.visres.2009.05.017 